\title{
Development of an ensemble machine learning prognostic model to predict 60-day risk of major adverse cardiac events in adults with chest pain
}

Chris J. Kennedy, $\mathrm{PhD}^{1,2,3,}{ }^{*}$; Dustin G. Mark, $\mathrm{MD}^{1}$; Jie Huang, $\mathrm{PhD}^{1}$; Mark J. van der Laan, $\mathrm{PhD}^{4}$; Alan E. Hubbard, $\mathrm{PhD}^{4}$; Mary E. Reed, $\mathrm{DrPH}^{1}$

1. Kaiser Permanente Northern California, Oakland, CA, USA

2. Department of Biomedical Informatics, Harvard Medical School, Boston, MA, USA

3. Department of Surgery, Beth Israel Deaconess Medical Center, Boston, MA, USA

4. Division of Biostatistics, UC Berkeley, Berkeley, CA, USA

*chris_kennedy@hms.harvard.edu

Version: August 19, 2021

\begin{abstract}
Background: Chest pain is the second leading reason for emergency department (ED) visits and is commonly identified as a leading driver of low-value health care. Accurate identification of patients at low risk of major adverse cardiac events (MACE) is important to improve resource allocation and reduce over-treatment.
\end{abstract}

Objectives: We assessed machine learning (ML) methods and electronic health record (EHR) covariate collection for MACE prediction. We aimed to maximize the pool of low-risk patients that were accurately predicted to have less than $0.5 \%$ MACE risk and could be eligible for reduced testing ("rule-out" strategy).

Population Studied: 116,764 adult patients presenting with chest pain in the ED between 2013 and 2015 and evaluated for potential acute coronary syndrome (ACS). 60-day MACE rate was 2\%.

Setting: Data analysis was performed May 2018 to August 2021.

Methods: We evaluated ML algorithms (lasso, splines, random forest, extreme gradient boosting, Bayesian additive regression trees) and SuperLearner stacked ensembling. We tuned ML hyperparameters through nested ensembling, and imputed missing values with generalized low-rank models (GLRM). Performance was benchmarked against individual biomarkers, validated clinical risk scores, decision trees, and logistic regression. We assessed clinical utility through net benefit analysis and explained the models through variable importance ranking and accumulated local effect visualization.

Results: The SuperLearner ensemble provided the best cross-validated discrimination with areas under the curve of 0.15 for precision-recall (PR-AUC) and 0.87 for receiver operating characteristic (ROC-AUC), and the best accuracy with an index of prediction accuracy of 0.07 . The ensemble's risk estimates were miscalibrated by 0.2 percentage points on average, and dominated the net benefit analysis at all examined thresholds. At a $0.5 \%$ threshold the ensemble model yielded 31

benefit-adjusted workups avoided per 100 patients, compared to 25 for logistic regression and 2-14 for clinical risk scores. The most important predictors were age, troponin, clinical risk scores, and electrocardiogram. GLRM achieved a $90 \%$ average reduction in reconstruction error compared to median-mode imputation.

Conclusion: Combining ML algorithms with a broad set of EHR covariates improved MACE risk prediction and would reduce over-treatment compared to simpler alternatives, while providing calibrated predictions and interpretability. Patients should receive targeted benefit in their care from thorough detection of nuanced health patterns via ML. 


\begin{abstract}
The omission of prediction from the major goals of basic medical science has impoverished the intellectual content of clinical work, since a modern clinician's main challenge in the care of patients is to make predictions.
\end{abstract}

Alvan Feinstein, 1983

\section{Introduction}

Chest pain is the second leading reason for emergency department visits (Rui et al. 2016) and is commonly identified as a leading driver of low-value health care (Sabbatini et al. 2014). Workup protocols in patients with chest pain are designed to diagnose the potential for major adverse cardiac events (MACE) (Kontos et al. 2010). Missed diagnoses of MACE can be cause for medico-legal action, which may encourage conservative testing without health benefit. Accurate identification of patients at low risk of MACE is important to improve resource allocation and reduce overtreatment (Amsterdam et al. 2010). Risk scores aim to identify patients eligible for early discharge (i.e. a "rule-out" strategy), avoiding additional strefss testing and cardiac imaging that is unlikely to be of benefit (Greenslade et al. 2018). The primary biomarkers used for initial triage are elevated cardiac troponin, a sensitive marker of cardiac injury measured serially, and repeated electrocardiograms (Scirica 2010; Smith et al. 2015).

Previous work has focused on the development and validation of additive risk scores as decision aids for risk stratification. Such risk scores examine a small number of biomarkers and demographics, summarize those predictors into qualitative levels, and use a weighted sum to allocate patients into risk categories. Standard risk scores are HEART (History, ECG, Age, Risk factors and Troponin) and EDACS (Emergency Department Assessment of Chest Pain Score - Than, Flaws, et al. 2014). HEART is most commonly used in North America, although EDACS has similar performance characteristics (Mark et al. 2018). Effective risk scores will stratify patients across risk levels such that the qualitative "low risk" group will have sufficiently low risk of short-term MACE that those patients can be discharged without additional workup. An ineffective or ill-calibrated risk score would underestimate the risk in the "low risk" group and lead to an overly optimistic early discharge policy that results in misidentified future MACE. But given multiple risk scores that are well-calibrated, scores with improved discrimination could theoretically result in a larger percentage of low-risk patients.

\subsection{Background and Objectives}

Building on Mark et al. (ibid.), we sought to assess the performance of machine learning (ML) methods at predicting MACE among emergency department patients with chest pain. We hypothesized that ML could improve upon existing validated risk scores through a more complex integration of predictors that can better estimate MACE risk. Our clinical objective was to maximize the pool of low-risk patients that are accurately predicted to have less than $0.5 \%$ MACE risk and may be eligible for reduced testing. The primary threshold of $0.5 \%$ risk has previously been identified as an acceptable risk by a majority of emergency physicians for early discharge (Than, Herbert, et al. 2013). Using a risk of $0.5 \%$ as the test threshold will inherently lead to a negative predictive value of greater than $99.5 \%$, provided that the risk prediction is well-calibrated in the target population. We also examined secondary thresholds of $1.0 \%$ and $2.0 \%$ given varying risk tolerance thresholds among practicing emergency physicians (ibid.), as well as presumed variation in patient risk preferences.

A reasonable assessment of ML performance could only be made in comparison to realistic alternative options. We compared ML performance to simpler indicators of risk: key biomarkers (troponin, electrocardiogram), validated clinical risk scores (History, ECG, Age, Risk factors and Troponin [HEART] and Emergency Department Assessment of Chest pain Score [EDACS]), as well as simpler models: decision trees and logistic regression.

If machine learning can demonstrate improved discriminative performance compared to logistic regression and related methods, along with appropriate calibration, its next hurdle for adoption is to provide interpretability. Clinicians may be willing to forgo maximum predictive accuracy for the sake of understanding how individual predictors influence the output of the algorithm. With analytical 
effort it may be possible to provide sufficient interpretability for clinicians to accept the complication of machine learning and the benefit of the (potentially) improved predictive accuracy. To facilitate interpretation, we explained the models through prediction-based variable importance ranking and accumulated local effect visualization. If simpler algorithms remain preferred, the ML results can at least approximately the best achievable performance, and so serve as benchmark standards when considering more restrictive algorithms.

\section{Data and Methods}

\subsection{Source of data}

Our study was sourced from the electronic health record (EHR) of 21 emergency departments (EDs) within Kaiser Permanente Northern California, an integrated health care delivery system with over 1 million annual ED visits.

\subsection{Participants}

All adult patients were retrospectively included if they had received cardiac troponin testing in the emergency department between 2013 and 2015 and either presented with a chief complaint of chest pain or chest discomfort, or whose ED physician had assigned them a primary or secondary ICD-coded diagnosis of chest pain. The later inclusion criterion is important because patients may complain of "anginal equivalents" (such as shortness of breath) in lieu of overt chest pain (Amsterdam et al. 2010). The initial inclusion pool had a 60 -day MACE rate of $8.0 \%$. Patients were excluded if they had a MACE diagnosis in the ED or within 30 days prior to ED visit, alternative non-ACS diagnoses at index visit (e.g. pneumonia, pneumothorax, or traumatic injury), could not be tracked due to lack of active health plan membership during the study (except in cases of death), or had a troponin I >99th percentile upper limit of normal given the dominant predictive value of elevated troponin values for adverse outcomes in both patients with acute coronary syndromes and in the general population (Bonaca et al. 2010; De Lemos et al. 2010). Patients were excluded if their smoking status was unknown, which was viewed as a key marker of low-quality data. The final study cohort consisted of 116,764 patients with a 60 -day MACE incidence of $1.88 \%$. A fourth-generation troponin assay was used during the study period (AccuTnI +3 , Beckman-Couleter, Brea, CA, USA).

\subsection{Outcome}

Our primary outcome was cumulative MACE incidence within 60 days of the index visit. We defined MACE as myocardial infarction, cardiogenic shock, cardiac arrest, or death.

\subsection{Predictors}

We used a total of 74 predictors sourced from the electronic health record, including vitals, labs, history, qualitative interpretation of ECG imaging, regular expression-based extraction of features from clinical notes, demographics, and missingness indicators (20). These predictors are detailed in the supplemental information.

\subsection{Missing data}

Missingness rates for each predictor are listed in Supplemental Table 1. We created missingness indicators for each predictor with 1 or more missing values, which marked the observations that were missing a value. Inclusion of missingness indicators often improves predictive performance (Agor et al. 2019; Sperrin et al. 2020), in part because it can reflect the information-seeking behavior of clinicians stemming from medical diagnosis and evaluation (Agniel et al. 2018; Groenwold 2020; Sisk et al. 2021). The set of missingness indicators was analyzed for perfect collinearity, and duplicate indicators were dropped.

Missing predictor values were imputed by factorizing the raw data matrix with generalized low-rank models (GLRM) (Schuler et al. 2016; Udell et al. 2016), a generalization of principal component 
analysis and matrix completion methods designed for mixed type data frames (continuous, categorical, ordinal, or binary variables). GLRM decomposes the predictor data into a matrix of reduced components and matrix of archetypes, including possible penalty terms that can induce sparsity (L1) or simply denoise (L2 or quadratic). Multiplying these two matrices reconstructs the original data frame, imputing any data entries with missing values; it will also allow imputation of missing values in future observations, even for predictors with no missingness in the current dataset. Additional details are provided in the supplemental information. Multiple imputation was not necessary because our scientific goal was to characterize predictive performance for the unimputed outcome variable, rather than to estimate statistical parameters for covariates that were imputed, such as linear regression coefficients (Sisk et al. 2021; Sperrin et al. 2020).

GLRM imputation greatly increased the number of unique values (cardinality) for continuous variables, which would have a negative performance impact on tree-based algorithms that test every unique value for a potential split. To avoid that performance drop, we using penalized histogram binning to bin imputed predictors with high cardinality into up to 200 unique values (Rozenholc et al. 2010).

We evaluated the benefit of the more complex GLRM-based imputation by comparing the imputed value to the known value, among variables with missingness. The root mean-squared reconstruction error metric was calculated for each variable, and for both GLRM and median/mode imputation methods. We could then estimate the percentage improvement in reconstruction error for the GLRM imputation for each variable, and the average across all predictors with missingness.

\subsection{Prediction algorithms}

Our prediction algorithm selection strategy focused on well-known algorithms that have shown strong performance in prior research, including both linear and tree-based estimation. The tree-based algorithms were random forest (Breiman 2001), extreme gradient boosting (XGBoost) (Chen et al. 2016), and Bayesian additive regression trees (Chipman et al. 2010). The linear prediction algorithms were generalized additive models (T. J. Hastie et al. 1990) using thin plate splines (Wood 2003), and lasso (Tibshirani 1996).

Splines have shown competitive performance with tree-based algorithms in prior clinical prediction work due to their ability to identify non-linear, but smooth patterns (Austin 2007). The lasso algorithm (or its generalization the elastic net) is a helpful test of sparsity in the covariates, and a faster \& more nuanced variable selection method than best subset or stepwise selection (T. Hastie et al. 2017). Better performance for lasso compared to logistic regression would indicate that feature selection could be helpful for other algorithms, while equal performance could indicate that the extraction of predictors from the EHR was overly restrictive and should be broadened.

\subsection{Benchmarks}

When evaluating complex algorithms it is important to contextualize their performance by thoroughly comparing to simpler alternative approaches or benchmarks. If the benchmark algorithms can achieve similar performance then the extra complexity of the statistical machine learning algorithms may not be worthwhile. The improvement of a novel prediction method over standard benchmarks is known as the skill of the prediction method (Brier 1950; Murphy et al. 1977; F. Sanders 1963). In clinical prediction the primary alternatives to statistical machine learning are relatively inflexible fits, which include logistic regression, ordinary least squares, individual decision trees, and stratification on key clinical covariates. We tested each of these options, where key covariates were defined as peak troponin, qualitative ECG reading, EDACS score, and HEART score. As a complement to stratification on different subsets of key covariates, we also evaluated logistic regression and decision trees when restricted to these key predictors.

\subsection{SuperLearner stacked ensembling}

When comparing a variety of algorithms an initial choice is to use cross-validation to select the algorithm with the best out-of-sample performance. This honest evaluation procedure allows comparing simpler linear models to complex (e.g. tree-based) ones, such that the appropriate amount 
of flexibility is chosen for the given dataset. A more nuanced decision would be to consider a weighted average of multiple algorithms - creating a team of algorithms whose contribution to the prediction is based on optimizing out-of-sample performance. That is the nature of stacked ensembles (Breiman 1996; Wolpert 1992), sometimes referred to as the Super Learner algorithm (van der Laan, Polley, et al. 2007). Rather than restrict our prediction machine to a single algorithm, we created a weighted average across all tested algorithms, and estimated weights based on an optimization goal so that they minimize a chosen performance statistic on test data. This ensembling procedure has been proven to perform asymptotically as well or better than the individual estimators (ibid.). We chose to optimize the Brier score (i.e. mean-squared error) in our ensemble, using convex weights based on a non-negative least squares meta-learner. Optimizing on Brier score includes a focus on both discrimination and calibration for the ensemble (Murphy et al. 1977). A convex combination of algorithm weights ensures that predictions fall within the convex hull of the constituent learners, while also inducing sparsity - i.e. algorithms can have zero weight.

\subsection{Hyperparameter tuning}

Prediction algorithms often have multiple hyperparameter settings that adjust the estimation procedure in different ways. Those hyperparameters are not estimated from the data, but rather must be specified a priori by the analyst. While software implementations will typically provide a default value for each hyperparameter, there is no reason to believe that the default values are effective for the current dataset. Customizing the hyperparameter configuration to the current dataset can allow the algorithms to adapt to the available sample size, number of predictor variables, measurement error in the predictors, sparsity in predictor relevance, and correlation structure of the predictors.

Hyperparameters are often chosen by fitting the algorithm with different configurations and selecting the configuration that maximizes accuracy on held-out data, such as through cross-validation. The benefit of hyperparameter tuning is believed to vary by algorithm, which is referred to as the tunability of the algorithm (Probst, Boulesteix, et al. 2019). Random forest, for example, is believed to work well with default hyperparameters but also can benefit from hyperparameter tuning, particularly to reduce overfitting (Probst, Wright, et al. 2019; Segal et al. 2011).

Hyperparameter tuning is inherently a computationally intensive process, as it involves fitting the algorithms many different times, and varies based on the number of hyperparameters (dimensionality) as well as number of the unique values tested for each hyperparameter (resolution). Further complexity is involved if one considers that some hyperparameters may be more important than others for a given algorithm. Given the role of hyperparameters in modifying the performance of prediction algorithms, caution is warranted when generalizing algorithm performance characteristics from individual studies (e.g. algorithm X outperforms algorithm Y), particularly when hyperparameters are left at their default values and therefore are not customized to the given dataset.

We adopted nested ensembling as our hyperparameter tuning strategy. Much as using a weighted ensemble of different algorithms may be preferable to selecting the single best-performing algorithm, using a weighted ensemble of hyperparameter settings for a given algorithm may yield improved performance compared to selecting a single set of hyperparameters. With that concept in mind we created small grids of hyperparameter configurations and estimated a SuperLearner ensemble for a given algorithm in which the ensemble weights selected the hyperparameter settings that maximized out-of-sample performance. This ensemble of hyperparameter settings could potentially rely on a single configuration due to the sparsity induced by the convex combination, or the optimization could distribute the weighting across multiple configurations if such a weighting improved performance over a single selected configuration. Another benefit of the nested ensembling is that it limits the number of learners that are analyzed in the outer SuperLearner ensemble, which can increase stability in the meta-learning process (i.e. allocation of weights in the convex combination). We used the ensemble hyperparameter tuning approach for random forests, xgboost, and individual decision trees. Details are provided in the supplemental information.

\subsection{Evaluation}

We evaluated risk prediction models based on their discrimination, calibration, and clinical utility. Nested cross-validation with 5 folds was used to conduct the discrimination and calibration analyses. 
While bootstrap estimation has been promoted for evaluation of clinical prediction models (Austin and Tu 2004; Steyerberg, Harrell Jr, et al. 2001), recent work has shown that the bootstrap can be biased for evaluating the performance of highly adaptive ML algorithms estimators such as random forests (Benkeser et al. 2019).

\subsubsection{Discrimination}

We chose area under the precision-recall curve (PR-AUC, also known as average precision [AP]) as our primary performance metric for evaluating discrimination, because it highlights performance differences that may be obscured by ROC-AUC with imbalanced data (Cook 2007; Saito et al. 2015). The PR-AUC compares positive predictive value to sensitivity across thresholds, focusing on model performance for the rare positive MACE cases (Davis et al. 2006). We included area under the receiver operating characteristic curve (ROC-AUC or the concordance statistic) as our secondary performance metric, which remains highly popular and interpretable (Janssens et al. 2020). The downside of ROC-AUC is that its reliance on the false positive rate gives the model "credit" for predicting negative cases correctly (true negatives), which is not challenging due to the rarity of MACE. As an exploratory metric we also estimated the adjusted Brier score (index of prediction accuracy) which integrates discrimination and calibration into a single metric (Kattan et al. 2018). We visualized improvements in discriminative performance using density plots of the calibration slope (Steyerberg, Vickers, et al. 2010). We did not conduct a reclassification analysis due to recognized limitations (Hilden et al. 2014; Kerr et al. 2014; Leening et al. 2014; Pepe et al. 2015).

\subsubsection{Calibration}

Our clinical use case was centered on a risk threshold of $0.5 \%$ to classify patients as "low risk" in order to qualify for early discharge. Because of that scientific goal, it was especially important to compare the model's predicted risks to the observed risks, i.e. its calibration (Lichtenstein et al. 1981) - also known as reliability (Brier 1950; Murphy et al. 1977) or external correspondence (Yates 1982). We assessed the calibration of predicted probabilities in four ways: 1) analysis of the calibration slope of the predicted risk (Cox 1958; Steyerberg, Harrell Jr, et al. 2001), 2) calibration curve visualization with lowess-smoothing and decile groups, 3) calculating the average absolute value between the predicted risk and the lowess-smoothed observed risk, known as the integrated calibration index (Austin and Steyerberg 2019) and mean absolute prediction error (van Smeden et al. 2019), 4) calculation of the index of prediction accuracy (IPA), a transformation of the Brier score (Kattan et al. 2018). We did not conduct a Hosmer-Lemeshow group-based calibration test due to its recognized limitations and recommendations against its use (Kramer et al. 2007; Van Calster et al. 2019).

As a statistical complement to the visual examination, we also calculated mean absolute prediction error (MAPE). MAPE is the sample mean of the absolute difference between the smoothed observed risk and the predicted risk. This statistic depends on the span of data used to smooth the observed risk, therefore the span used should be reported and multiple span hyperparameter values should be considered, if not a continuous curve across reasonable span values.

$$
\frac{1}{n} \sum_{i=1}^{n} \mid \operatorname{Observed} \operatorname{Risk}(i)-\text { Predicted Risk }(i) \mid
$$

\subsubsection{Clinical utility}

Discrimination and calibration are important characteristics of model performance, but they do not directly capture the impact of the model on patient care when used for clinical decision support (Vickers, Van Calster, et al. 2016). The planned clinical use of the prediction model was first to assess eligibility for early discharge among low-risk patients. Accurately estimating the risk of MACE for patients would allow those low-risk patients to be discharged and avoid additional unnecessary workup (overtreatment) and would free up resources (clinical attention, testing capacity, etc.) for higher risk patients. Low risk was generally defined as being below a $0.5 \%$ well-calibrated probability of MACE within 60 days, with less conservative thresholds of $1 \%$ and $2 \%$ as additional options.

Our model needed to balance two trade-offs: 1) false negatives in which a patient was identified as low-risk but whose true risk was above the clinical threshold, and 2) false positives in which patients 
were believed to be above the given threshold but whose true risk was less than the threshold. Errors in the first category have a greater cost than those in the second category, because there is a greater potential detriment to those patients who were discharged early but whose true risk exceeded the threshold. Patients incorrectly estimated to be above the risk threshold, but who are truly low risk, have comparatively minor costs of additional workup, use of clinical resources, and potential to be overtreated.

This balancing of trade-offs based on a clinical decision threshold can be evaluated using net benefit analysis of decision curves (Vickers and Elkin 2006; Vickers, Van Calster, et al. 2016). The net benefit of a model or strategy at a given threshold is (Vickers and Elkin 2006):

$$
\text { Net benefit }=\text { True positives } / \mathrm{N}-\text { False positives } / \mathrm{N} \times\left(\frac{\text { threshold }}{1-\text { threshold }}\right)
$$

The net benefit formula reflects a relative weighting of true positives versus false positives, which is the threshold / ( 1 - threshold) term that scales the percentage of false positives. As has been described previously (ibid.), a particular decision threshold inherently embeds a relative weighting. In our case a threshold of $0.5 \%$ corresponds to a weight of $0.5 \% / 99.5 \%=0.00503$; if we take the inverse that means that a false negative (missed MACE) is 199 times as bad as a false positive (overtreatment). In contrast, a threshold of $2 \%$ implies that a missed MACE is only 49 times as bad as further workup for a patient who will not go on to have a MACE.

In a "rule out" scenario where a threshold is applied to a patient's estimated risk to determine eligibility for early discharge, one can compare benefit-adjusted net reduction in unnecessary interventions curves across clinically relevant decision thresholds, scaled to 100 patients for ease of use (ibid.):

$$
100 \times(\text { net benefit of the model }- \text { net benefit of treat all }) /\left(\frac{\text { threshold }}{1-\text { threshold }}\right)
$$

This form of net benefit analysis answers the question: relative to a naive strategy of treating all patients, how many patients can the risk model remove from treatment at a given cutoff threshold, after adjusting for the harm caused by some of those removed patients going on to have a MACE event (false negatives)?

\subsection{Interpretability}

Beyond the statistical performance of a clinical prediction, it can be important to provide an explanation or overview of how a model generates its predictions. Interpretation is desirable first because it can provide evidence that the model is working as expected, which can improve the trustworthiness of its predictions for clinicians, patients, or collaborators. Interpretation may also lead to scientific insights about how predictors are related to the outcome, which could be conceptualized as causal pathways, data generating processes, or biological mechanisms. Interpretation can further inform the data export and cleaning processes, such as identifying extreme values, data entry errors, or outliers, or suggesting additional predictor variables to incorporate into the model.

Methods of interpretation can be model-specific or model-agnostic. For models within the family of linear regression, one might provide the estimated beta coefficients for each predictor, along with their associated confidence intervals and p-values. Interpretation becomes less straightforward as models become more complex, such as with interaction or polynomial terms in a regression, random forest or boosted tree models with hundreds or thousands of non-linear decision trees, or splines in which ranges of a given predictor might have different coefficients.

In this work we focus on two complementary forms of model interpretability: variable predictive importance ranking and accumulated local effect plots, as described below.

\subsubsection{Variable predictive importance ranking}

Prediction-oriented variable importance rankings order the predictor variables by their contribution to a model's prediction, providing evidence as to which predictors were relied upon the most by the algorithm. Such rankings could be used as a form of confirmatory analysis if a hypothesized ranking were created prior to data analysis, which could formally identify predictors that differed from their expected importance. 


\subsubsection{Accumulated local effects}

It may also be helpful to understand how a model's prediction varies over the values of individual predictors, particularly continuous predictors with a wide range or large number of unique values. Partial dependence plots (PDPs) as proposed by Friedman (2001) are commonly used to provide this type of interpretability, but they can yield flawed results because they make a key unrealistic assumption that features are statistically independent of each other (Molnar 2020, p. 5.1.3).

Accumulated local effect (ALE) plots are a recently developed method that avoids that limitation of PDPs, by counterfactually modifying observations that lie within a nearby kernel neighborhood of the current predictor's value of interest (Apley et al. 2019). Following the variable importance ranking, we visualize the contribution of high-importance continuous variables using accumulated local effect plots.

\section{Results}

\subsection{Model performance}

\subsubsection{Discrimination}

Figure 1a displays the estimated precision-recall area under the curve (PR-AUC) PR-AUCs and 95\% confidence intervals for each combination of features and estimation algorithm. The MACE mean on the training sample represents the baseline PR-AUC, which was $1.88 \%$. The SuperLearner ensemble achieved the highest estimated PR-AUC $(0.148,95 \%$ CI $[0.126,0.170])$, followed by the random forest with hyperparameter tuning $(0.144,[0.125,0.164])$, the default random forest $(0.143,[0.122,0.165])$, and the tuned XGBoost $(0.138,[0.116,0.160])$. By comparison the PR-AUC for logistic regression was $0.120[0.103,0.137]$, noticeably lower than the ensemble. Point estimates and confidence intervals are listed in eTable 3.

For our secondary discrimination metric, cross-validated ROC-AUC was calculated and is displayed in Figure 1b (LeDell et al. 2015). The SuperLearner ensemble again achieved the highest performance $(\mathrm{ROC}-\mathrm{AUC}=0.866,95 \% \mathrm{CI}[0.859,0.873])$, followed by tuned random forest $(0.860,[0.853,0.867])$, BART $(0.859,[0.852,0.866])$, and tuned XGBoost $(0.859,[0.852,0.866])$. The ROC-AUC for logistic regression $(0.842,[0.834,0.850])$ was significantly lower than the ensemble. Point estimates and confidence intervals are listed in eTable 4.

Figure 1. Comparison of cross-validated discriminative performance using a) PR-AUC metric and b) ROC-AUC, with $95 \%$ confidence intervals.

A.

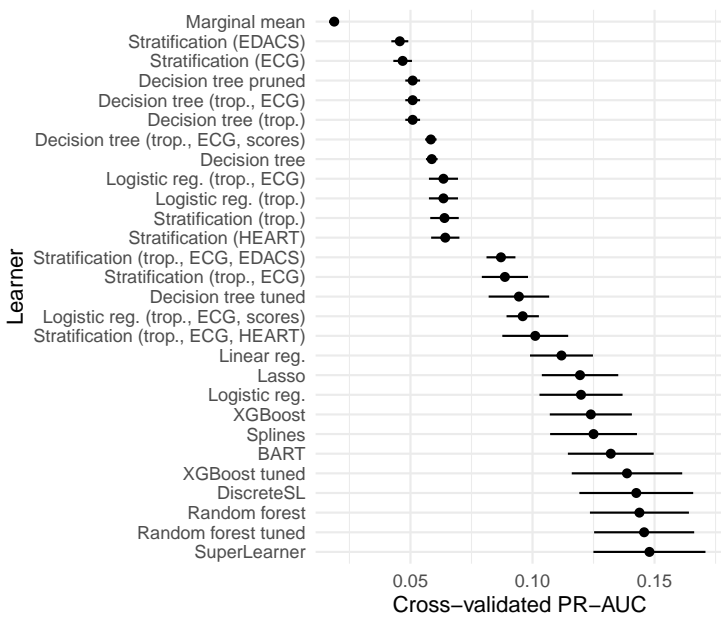

B.

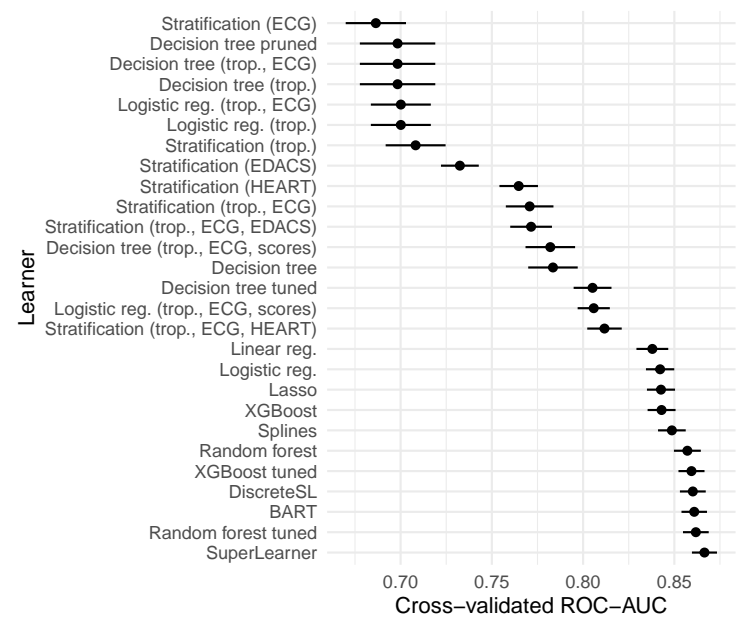

We reviewed the distribution of learner weights in the SuperLearner ensemble to examine which algorithms were used most heavily (eTable 2). Four algorithms were always incorporated into the ensemble: default random forest (average weight $=0.25$ ), tuned random forest (average weight $=0.25$ ), 
default XGBoost (average weight $=0.20$ ), and BART (average weight $=0.18$ ). The remaining 3 learners were sometimes incorporated into the ensemble, with average weights ranging from 0.08 to 0.004 and a maximum individual weight of 0.18 .

\subsubsection{Calibration}

We visually compared the predicted risk of the SuperLearner ensemble to the lowess-smoothed observed risk in Figure 2. The red line is the target calibration in which predicted risk is equal to observed risk. The blue line shows the lowess-smoothed observed risk for each value of the predicted risk. The median predicted risk was $0.64 \%$, with a first quartile of $0.2 \%$ and third quartile of $2 \%$. Our primary threshold of scientific interest was $0.5 \%$ for possible early discharge. Given those low risk levels, it would be best to "zoom in" our visual calibration review to that region. We show a zoomed calibration plot as Figure 2a.

We further include a exponential-scale calibration plot (Figure $2 \mathrm{~b}$ ) with calibration confidence intervals after grouping patients into 10 groups based on predicted risk, consistent with TRIPOD guideline recommendations (Collins et al. 2015). Due to the low rate of MACE the exponential scaling of axes allows easier comparison across the probability range, although it may be less visually intuitive due to the shifting of scales.

Figure 2. Calibration plot comparing predicted risk to observed risk for the ensemble model. Clinical thresholds of $0.5 \%, 1 \%$, or $2 \%$ risk are noted by blue vertical lines. A) linear scale, b) log scale.

A.

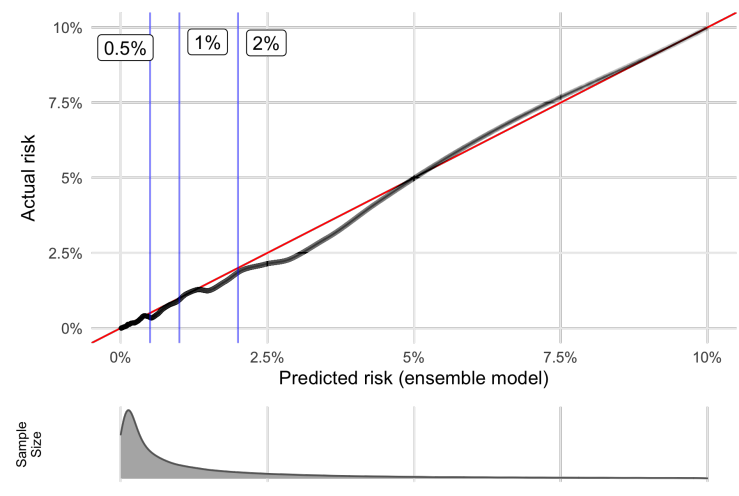

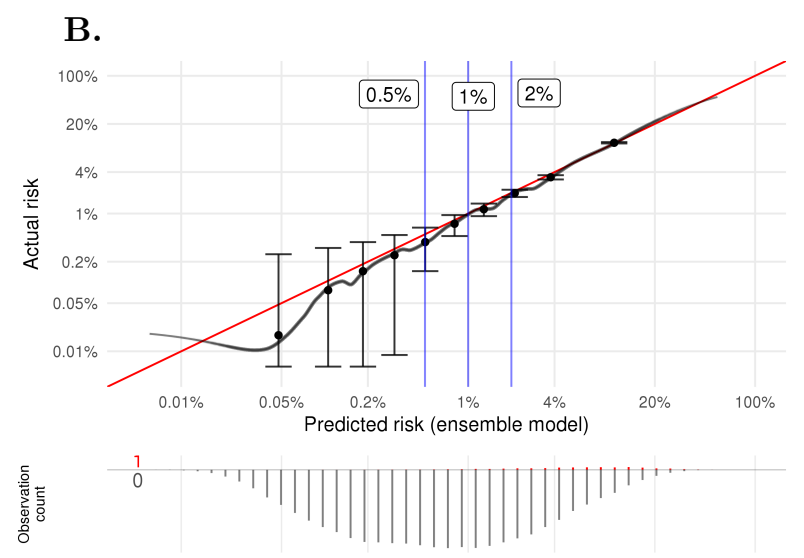

B.

We found a mean absolute prediction error (MAPE) of $0.19 \%$ with a lowess smoothing span of 0.05 (low smoothing), and an MAPE of $0.14 \%$ with a smoothing span of 0.20 (moderate smoothing). These statistics indicate that the ensemble risk prediction was typically miscalibrated by about 0.17 percentage points.

\subsubsection{Clinical utility}

The ensemble model dominated the net reduction in interventions curves across all clinically relevant decision thresholds between $0 \%$ and $4 \%$ (Figure 3). This finding provides conclusive, direct evidence that the ensemble model would lead to the best patient care among the evaluated options, and is therefore an ethical imperative to bring into clinical practice. The relative improvement for the ensemble model, as well as random forest and logistic regression, was particularly high at conservative decision thresholds between $0.6 \%$ and $0 \%$, when compared to the risk scores or a single decision tree. 
Figure 3. Clinical utility evaluation via benefit-adjusted net reduction in unnecessary interventions per 100 patients.

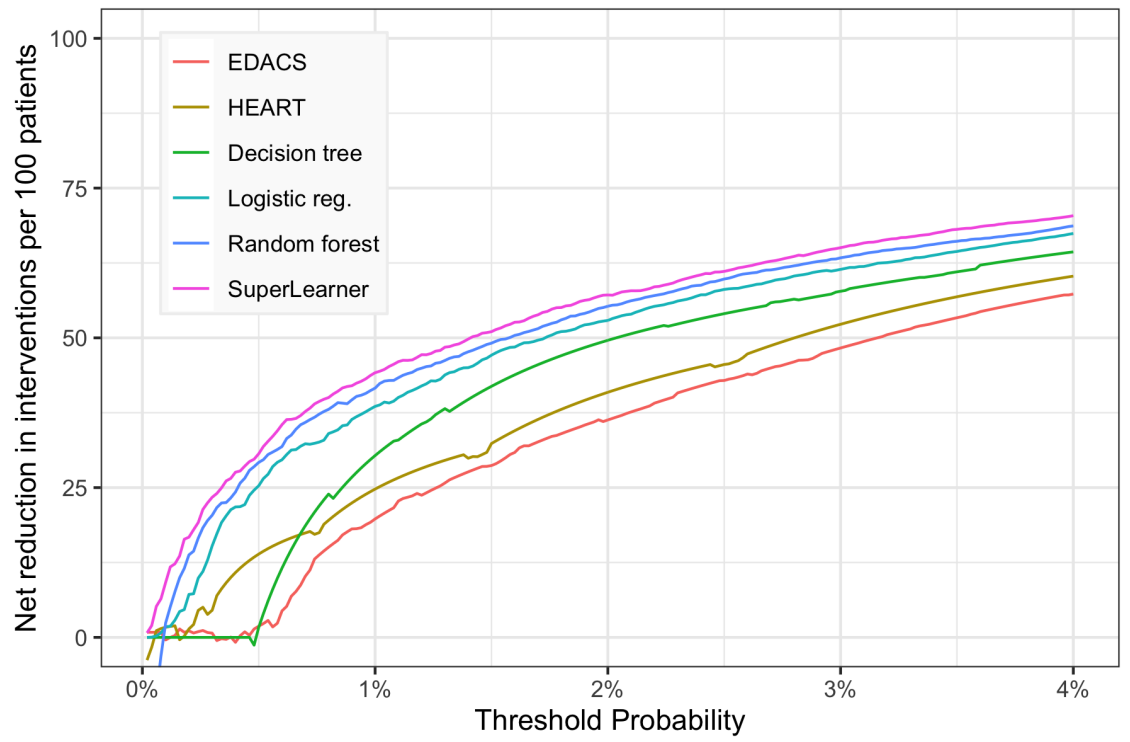

\subsubsection{Missing data imputation}

Compared to median/mode imputation, GLRM-based imputation yielded an average reduction in reconstruction error of $90 \%$ (eTable 5), suggesting a beneficial ability to capture missingness patterns.

\section{$3.2 \quad$ Interpretation}

\subsubsection{Variable importance ranking}

As discussed earlier, our objective for the variable importance analysis was to understand which variables were most influential on the prediction of our final model. Providing that ranking could improve the interpretability of the risk prediction, allowing for confirmation that the results are reasonable and possibly yielding additional scientific insights. However, our final model is quite complex: it is a weighted average of multiple versions of random forests, xgboost models, bayesian additive regression trees, etc. In this work we provide rankings for the top two estimation algorithms: random forest and xgboost (Table 1). We used the optimal hyperparameter settings from cross-validated analysis.

Interestingly we see rather different results between the two algorithms, which supports the hypothesis than an ensemble of multiple algorithms could achieve better performance than selecting a single estimation algorithm. Both algorithms place high emphasis on the EDACS and HEART risk scores, demonstrating the benefit of including those scores along with the underlying predictors. Different versions of the cardiac troponin predictor are emphasized by the two algorithms: random forest focuses on 3-hour troponin whereas xgboost focuses on peak troponin. ECG reading is emphasized by xgboost but not random forest. Both algorithms make use of lipid profile predictors (LDL, HDL) and vital signs (pulse, respiration) that are not included in the existing risk scores. The random forest makes use of missingness indicators from the elements of a lipid panel, while xgboost does not. Also noteworthy is the lack of pain-related characteristics sourced from clinical notes in the top predictors, a difference from prior MACE prediction work (Amsterdam et al. 2010).

\subsubsection{Accumulated local effects}

Based on the rankings from the variable importance analyses, we generated accumulated local effect plots (Figure 4) to visualize the conditional relationship of top predictors to the ensemble's prediction, across their range of values. 
Table 1. Variable importance rankings

A. Random Forest importance ranking

\begin{tabular}{lr}
\hline \multicolumn{1}{c}{ Variable } & \multicolumn{1}{c}{$\begin{array}{c}\text { Mean } \\
\text { Decrease } \\
\text { Accuracy (\%) }\end{array}$} \\
\hline 1. Age & 0.262 \\
2. EDACS & 0.188 \\
3. HEART & 0.117 \\
4. CAD & 0.117 \\
5. Troponin 3HV & 0.111 \\
6. LDL & 0.104 \\
7. Total Cholesterol & 0.102 \\
8. Missing Triglcyerides & 0.083 \\
9. Missing Total Cholesterol & 0.080 \\
10. Missing LDL & 0.076 \\
11. Missing HDL & 0.073 \\
12. Pulse & 0.071 \\
13. Peak pulse & 0.070 \\
14. BMI & 0.051 \\
15. Diabetes & 0.047 \\
16. Hypertension & 0.045 \\
17. HDL & 0.044 \\
18. HbA1c & 0.043 \\
19. Peak troponin & 0.041 \\
20. Triglycerides & 0.039 \\
\hline
\end{tabular}

B. XGBoost importance ranking

\begin{tabular}{lc}
\hline \multicolumn{1}{c}{ Variable } & Gain \\
& \\
\hline 1. Peak troponin & 0.3288 \\
2. HEART & 0.1591 \\
3. High EDACS & 0.0712 \\
4. EDACS & 0.0457 \\
5. High HEART & 0.0444 \\
6. ECG & 0.0415 \\
7. Peak pulse & 0.0320 \\
8. Age & 0.0282 \\
9. BMI & 0.0234 \\
10. SBP & 0.0217 \\
11. Myocardial infarction & 0.0210 \\
12. CAD & 0.0198 \\
13. Aortic athero. & 0.0175 \\
14. Troponin 3HV & 0.0165 \\
15. HDL & 0.0159 \\
16. Respiration & 0.0135 \\
17. O2 saturation & 0.0131 \\
18. GFR & 0.0130 \\
19. Exertion & 0.0127 \\
20. Lowest SBP & 0.0091 \\
\hline
\end{tabular}

\section{Discussion}

It remains debated whether machine learning methods can exhibit statistically and substantively significant benefits for risk prediction compared to logistic regression, decision trees, or additive risk scores (Goldstein, Navar, and Carter 2016; Goldstein, Navar, Pencina, et al. 2016; R. Khera et al. 2021). A recent meta-analysis, for example, did not find systematic benefit from machine learning in comparison to logistic regression (Christodouloua et al. 2019). Yet there is also optimism about the potential for artificial intelligence methods in medicine (He et al. 2019) in general, as well as cardiology specifically (Johnson et al. 2018). As long as cross-validation is used as a neutral evaluation procedure, the debate is unnecessary because we can test and select the algorithm(s) with the appropriate amount of flexibility (bias-variance trade-off) for the signal in the current dataset (van der Laan and Dudoit 2003).

Certain analytical characteristics would be important to arrange in order for ML to potentially improve upon simpler options. First, it was important to extract a broader set of granular predictor variables than were used by existing scores. Extensive predictor sets give ML the potential to capture multi-variable interactions and nonlinear relationships that are missed by linear or additive approaches, perhaps relevant only to certain subgroups of patients. Further, ML may statistically identify novel predictors that have been missed by existing scores or the broader literature, or whose predictive impact was too small, in too complex a form, or underrepresented in terms of sample size to be detected by non-ML methods. The expansion of electronic health records (EHRs) also makes broader covariate collection more feasible and relevant than was possible prior to EHRs, while also facilitating more granular measurement of variables (E. H. Kennedy et al. 2013).

It is also helpful for variables be measured on a fine-grained scale, which gives ML the opportunity to detect novel cut-points or thresholds that improve performance. Variables should be kept as their original continuous measurements rather than dichotomized or discretized into qualitative levels (Senn 
Figure 4. Accumulated local effect plots of key continuous predictors.

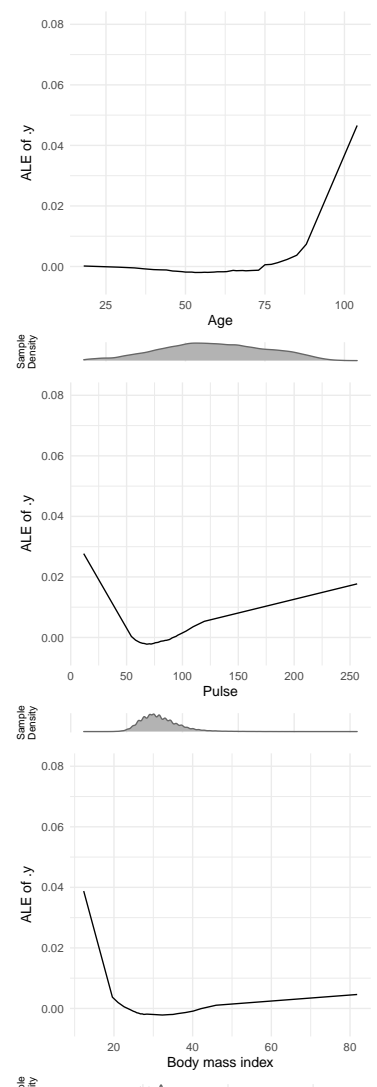

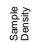
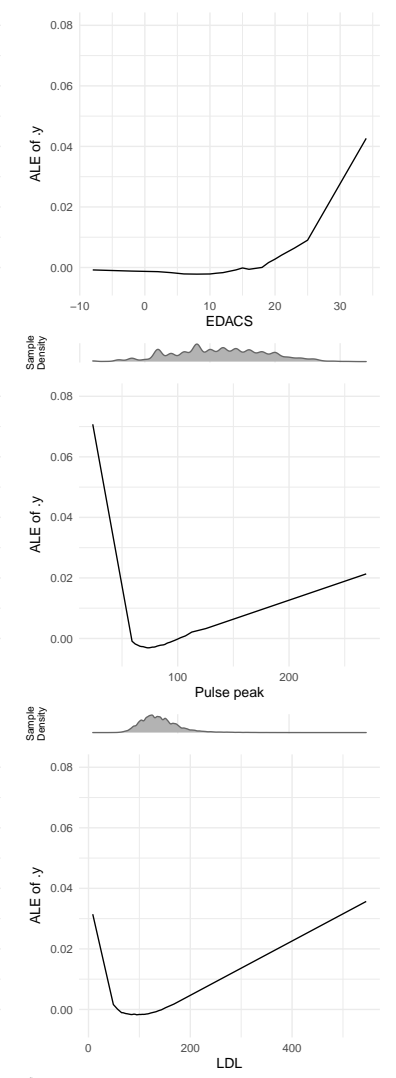

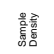
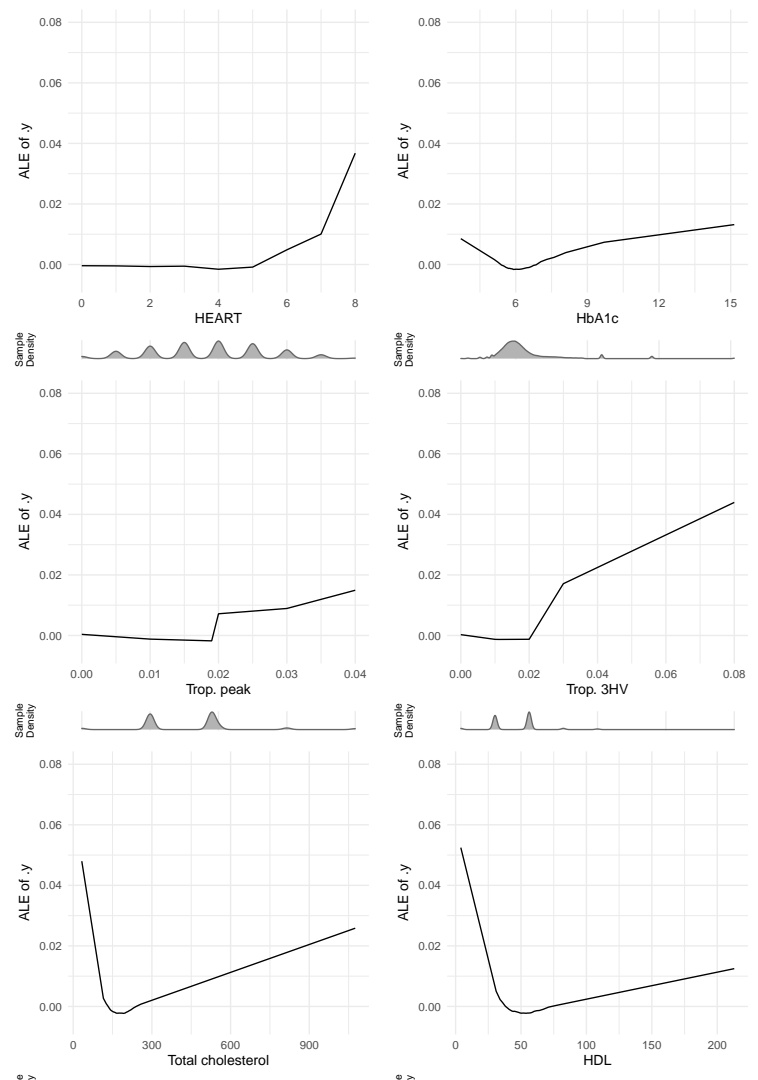

量高
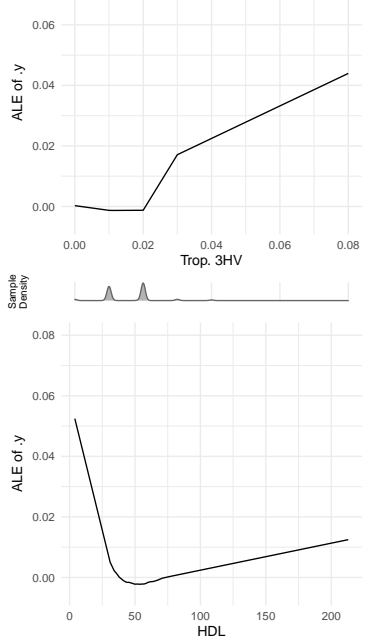

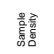

2005). For example, a predictor such as body mass index (BMI) loses substantial information when it is dichotomized into an indicator of high-BMI or the absence of high-BMI. A single threshold chosen for for that dichotomization may not be optimal for certain subgroups or regions of risk; for example, low BMI is often a stronger risk factor of disease than obesity. One of the benefits of ML is that it can identify thresholds in a data-adaptive way, allowing it to better approximate unknown or ill-understood physiological processes. That said, very high cardinality variables can result in overfitting and slow down the training of certain machine learning algorithms, such as decision trees, that test each unique value as a possible subgroup splitting threshold. It may be beneficial to reduce the cardinality of granular continuous variables through histogram binning that scales with the dataset size, e.g. $\log ($ sample size $) \times 5$.

\subsection{Limitations and future work}

The next step in model evaluation is to conduct one or more external validations of the discrimination, calibration, and net benefit of the ensemble model (Moons et al. 2012). This validation might include future retrospective or prospective cohorts at the current study location (temporal validation), although preferably cohorts sourced from other regions or EHRs (geographical or institutional validation) (ibid.). We hope to collaborate with groups interested in such validations.

We aim to expand the machine learning in several ways in future work. Cross-validation could be conducted using temporal splitting to incorporate loss in performance due to distribution changes over time (Roberts et al. 2017). Additional machine learning algorithms could be tested for benefit, such as LightGBM, CatBoost, extremely randomized trees, multivariate adaptive regression splines, and deep learning for tabular data. The ensemble weighting (metalearner) could directly optimize net benefit. Incorporating feature ranking and selection may benefit the simpler algorithms by removing unhelpful predictors, such as through ensemble methods (Effrosynidis et al. 2021). Feature engineering might be beneficial as well, such as creation of interaction terms or even incorporation of the principal 
components from the GLRM imputation. Due to computational limitations we were not able to conduct hyperparameter tuning on the BART learner, which likely would provide some performance benefit. We are optimistic that random search or model-based search (e.g. Hyperband) rather than grid search could provide even stronger tuning of algorithm hyperparameters across a higher number of dimensions ( $\mathrm{Li}$ et al. 2017). Imputation model training and data standardization could be restricted to the training fold, avoiding even unsupervised use of test set data (Jaeger et al. 2020). Evaluation of the GLRM imputation could be further contextualized through cross-validated comparisons to additional imputation methods, especially principal component analysis, k-nearest neighbors, and deep autoencoders, or even stacked ensemble imputation strategies. The variable importance ranking could be extended through a random forest-style permutation importance analysis of the ensemble model, conditional importance measures (Strobl et al. 2007), and through targeted learning methods such as vimp (Williamson et al. 2017) or varimpact (Hubbard et al. 2018). Calibration might be improved through targeted learning-based adjustment (Brooks et al. 2012) or isotonic regression on validation data. Cross-validated estimation of discrimination performance could be improved through cross-validated targeted maximum likelihood estimation (Benkeser et al. 2019).

Beyond those technical considerations, bringing this technology to the bedside is a remaining task. Implementing this work as a clinical decision support tool for real-time usage would require extracting and preparing the predictor variables for a given patient (including possible in-clinic data entry for recently acquired predictors), running the model to generate the prediction, and then visualizing or otherwise returning the prediction for usage by medical providers (Sendak et al. 2020). Stakeholder engagement, including user testing, training, and feedback, would be necessary to ensure widespread adoption of the tool (Scheinker et al. 2020).

\section{Conclusion}

In this work we explored the benefit of complex machine learning algorithms for predicting major adverse cardiac events in patients with chest pain. We found that the ML algorithms were able to achieve improved discrimination compared to simpler baselines such as logistic regression, decision trees, or stratification on individual predictors. Combining multiple algorithms into an ensemble estimator yielded the best performance, and rather than select optimal hyperparameters we created an ensemble of algorithms across different hyperparameters. We showed the surprising effectiveness of generalized low-rank models for imputation of missingness in EHR-sourced patient data. The result was a well-calibrated ensemble model that dominated patient net benefit analysis at all examined risk thresholds, demonstrating the utility for patient care as a clinical decision support system. Finally, we provided interpretation of how the ensemble's prediction is generated through two methods: ranking the predictors by their contribution to predictive performance, and visualizing the dose-response effect of continuous predictors with accumulated local effect plots.

\section{Funding}

This work was supported by a Kaiser Permanente Division of Research Delivery Science research grant.

\section{Acknowledgments}

We thank Dustin Ballard, Gabriel Escobar, Alan Ho, Oleg Sofrygin, and Jodi McCloskey for helpful comments, and Adina Rauchwerger and Laura Simon for project management. The software code for this project has been translated to use a public dataset and is available online at https://github.com/ck37/Predictive-Modeling-in-R, with supporting functions in the open source R package ck37r (C. J. Kennedy 2020).

\section{References}

Agniel, Denis, Isaac S Kohane, and Griffin M Weber (2018). "Biases in electronic health record data due to processes within the healthcare system: retrospective observational study". In: Bmj 361. 
Agor, Joseph, Osman Y Özaltın, Julie S Ivy, Muge Capan, Ryan Arnold, and Santiago Romero (2019). "The value of missing information in severity of illness score development". In: Journal of biomedical informatics 97, p. 103255.

Amsterdam, Ezra A, J Douglas Kirk, David A Bluemke, Deborah Diercks, Michael E Farkouh, J Lee Garvey, Michael C Kontos, James McCord, Todd D Miller, Anthony Morise, et al. (2010). "Testing of low-risk patients presenting to the emergency department with chest pain: a scientific statement from the American Heart Association". In: Circulation 122.17, pp. 1756-1776.

Apley, Daniel W and Jingyu Zhu (2019). "Visualizing the effects of predictor variables in black box supervised learning models". In: arXiv preprint arXiv:1612.08468.

Austin, Peter C (2007). "A comparison of regression trees, logistic regression, generalized additive models, and multivariate adaptive regression splines for predicting AMI mortality". In: Statistics in medicine 26.15, pp. 2937-2957.

Austin, Peter C and Ewout W Steyerberg (2019). "The Integrated Calibration Index (ICI) and related metrics for quantifying the calibration of logistic regression models". In: Statistics in medicine 38.21, pp. 4051-4065.

Austin, Peter C and Jack V Tu (2004). "Bootstrap methods for developing predictive models". In: The American Statistician 58.2, pp. 131-137.

Benkeser, David, Maya Petersen, and Mark J van der Laan (2019). "Improved small-sample estimation of nonlinear cross-validated prediction metrics". In: Journal of the American Statistical Association, pp. 1-16.

Bonaca, Marc, Benjamin Scirica, Marc Sabatine, Anthony Dalby, Jindrich Spinar, Sabina A Murphy, Peter Jarolim, Eugene Braunwald, and David A Morrow (2010). "Prospective evaluation of the prognostic implications of improved assay performance with a sensitive assay for cardiac troponin I". In: Journal of the American College of Cardiology 55.19, pp. 2118-2124.

Breiman, Leo (1996). "Stacked regressions". In: Machine learning 24.1, pp. 49-64.

- (2001). "Random forests". In: Machine learning 45.1, pp. 5-32.

Brier, Glenn W (1950). "Verification of forecasts expressed in terms of probability". In: Monthly weather review 78.1, pp. 1-3.

Brooks, Jordan, Mark J van der Laan, and Alan S Go (2012). "Targeted maximum likelihood estimation for prediction calibration". In: The international journal of biostatistics 8.1.

Chen, Tianqi and Carlos Guestrin (2016). "Xgboost: A scalable tree boosting system". In: Proceedings of the 22nd acm sigkdd international conference on knowledge discovery and data mining, pp. 785-794.

Chipman, Hugh A, Edward I George, Robert E McCulloch, et al. (2010). "BART: Bayesian additive regression trees". In: The Annals of Applied Statistics 4.1, pp. 266-298.

Christodouloua, Evangelia, MA Jie, Gary S Collins, Ewout W Steyerberg, Jan Y Verbakel, Ben van Calster, et al. (2019). "A systematic review shows no performance benefit of machine learning over logistic regression for clinical prediction models". In: Journal of clinical epidemiology.

Collins, Gary S, Johannes B Reitsma, Douglas G Altman, and Karel GM Moons (2015). "Transparent reporting of a multivariable prediction model for individual prognosis or diagnosis (TRIPOD): the TRIPOD statement". In: British Journal of Surgery 102.3, pp. 148-158.

Cook, Nancy R (2007). "Use and misuse of the receiver operating characteristic curve in risk prediction". In: Circulation 115.7, pp. 928-935.

Cox, David R (1958). "Two further applications of a model for binary regression". In: Biometrika 45.3/4, pp. $562-565$.

Davis, Jesse and Mark Goadrich (2006). "The relationship between Precision-Recall and ROC curves". In: Proceedings of the 23rd international conference on Machine learning, pp. 233-240.

De Lemos, James A, Mark H Drazner, Torbjorn Omland, Colby R Ayers, Amit Khera, Anand Rohatgi, Ibrahim Hashim, Jarett D Berry, Sandeep R Das, David A Morrow, et al. (2010). "Association of troponin $\mathrm{T}$ detected with a highly sensitive assay and cardiac structure and mortality risk in the general population". In: Jama 304.22, pp. 2503-2512.

Effrosynidis, Dimitrios and Avi Arampatzis (2021). "An evaluation of feature selection methods for environmental data". In: Ecological Informatics 61, p. 101224.

Friedman, Jerome H (2001). "Greedy function approximation: a gradient boosting machine". In: Annals of statistics, pp. 1189-1232.

Goldstein, Benjamin A, Ann Marie Navar, and Rickey E. Carter (2016). "Moving beyond regression techniques in cardiovascular risk prediction: applying machine learning to address analytic challenges". In: European Heart Journal 38.23, pp. 1805-1814. ISSN: 0195-668X. DOI: 10.1093/eurheartj/ehw302. 
Goldstein, Benjamin A, Ann Marie Navar, Michael J Pencina, and John P A Ioannidis (2016). "Opportunities and challenges in developing risk prediction models with electronic health records data: a systematic review". In: Journal of the American Medical Informatics Association 24.1, pp. 198-208. IsSN: 1067-5027. DOI: $10.1093 /$ jamia/ocw042.

Greenslade, Jaimi H, Edward W Carlton, Christopher Van Hise, Elizabeth Cho, Tracey Hawkins, William A Parsonage, Jillian Tate, Jacobus Ungerer, and Louise Cullen (2018). "Diagnostic accuracy of a new high-sensitivity troponin I assay and five accelerated diagnostic pathways for ruling out acute myocardial infarction and acute coronary syndrome". In: Annals of emergency medicine 71.4, pp. 439-451.

Groenwold, Rolf HH (2020). "Informative missingness in electronic health record systems: the curse of knowing". In: Diagnostic and prognostic research 4.1, pp. 1-6.

Hastie, Trevor J and Robert J Tibshirani (1990). Generalized additive models. Vol. 43. CRC press.

Hastie, Trevor, Robert Tibshirani, and Ryan J Tibshirani (2017). "Extended comparisons of best subset selection, forward stepwise selection, and the lasso". In: arXiv preprint arXiv:1707.08692.

He, Jianxing, Sally L Baxter, Jie Xu, Jiming Xu, Xingtao Zhou, and Kang Zhang (2019). "The practical implementation of artificial intelligence technologies in medicine". In: Nature medicine 25.1, p. 30.

Hilden, Jørgen and Thomas A Gerds (2014). "A note on the evaluation of novel biomarkers: do not rely on integrated discrimination improvement and net reclassification index". In: Statistics in medicine 33.19, pp. 3405-3414.

Hubbard, Alan E, Chris J Kennedy, and Mark J van der Laan (2018). "Data-Adaptive Target Parameters". In: Targeted Learning in Data Science. Springer, pp. 125-142.

Jaeger, Byron C, Nicholas J Tierney, and Noah R Simon (2020). "When to Impute? Imputation before and during cross-validation". In: arXiv preprint arXiv:2010.00718.

Janssens, A Cecile J W and Forike K Martens (2020). "Reflection on modern methods: Revisiting the area under the ROC Curve". In: International Journal of Epidemiology. dyz274. ISSN: 0300-5771. DOI: 10.1093/ije/dyz274.

Johnson, Kipp W, Jessica Torres Soto, Benjamin S Glicksberg, Khader Shameer, Riccardo Miotto, Mohsin Ali, Euan Ashley, and Joel T Dudley (2018). "Artificial intelligence in cardiology". In: Journal of the American College of Cardiology 71.23, pp. 2668-2679.

Kattan, Michael W and Thomas A Gerds (2018). "The index of prediction accuracy: an intuitive measure useful for evaluating risk prediction models". In: Diagnostic and prognostic research 2.1, p. 7 .

Kennedy, Chris J (2020). ck37r: Chris Kennedy's $R$ toolkit. URL: https://github.com/ck37/ck37r.

Kennedy, Edward H, Wyndy L Wiitala, Rodney A Hayward, and Jeremy B Sussman (2013). "Improved cardiovascular risk prediction using nonparametric regression and electronic health record data". In: Medical care 51.3, p. 251.

Kerr, Kathleen F, Zheyu Wang, Holly Janes, Robyn L McClelland, Bruce M Psaty, and Margaret S Pepe (2014). "Net reclassification indices for evaluating risk-prediction instruments: a critical review". In: Epidemiology (Cambridge, Mass.) 25.1, p. 114.

Khera, Rohan, Julian Haimovich, Nathan C Hurley, Robert McNamara, John A Spertus, Nihar Desai, John S Rumsfeld, Frederick A Masoudi, Chenxi Huang, Sharon-Lise Normand, et al. (2021). "Use of machine learning models to predict death after acute myocardial infarction". In: JAMA cardiology.

Kontos, Michael C, Deborah B Diercks, and J Douglas Kirk (2010). "Emergency department and office-based evaluation of patients with chest pain". In: Mayo Clinic Proceedings. Vol. 85. 3. Elsevier, pp. 284-299.

Kramer, Andrew A and Jack E Zimmerman (2007). "Assessing the calibration of mortality benchmarks in critical care: The Hosmer-Lemeshow test revisited". In: Critical care medicine 35.9, pp. 2052-2056.

LeDell, Erin, Maya Petersen, and Mark van der Laan (2015). "Computationally efficient confidence intervals for cross-validated area under the ROC curve estimates". In: Electronic journal of statistics 9.1, p. 1583.

Leening, Maarten JG, Moniek M Vedder, Jacqueline CM Witteman, Michael J Pencina, and Ewout W Steyerberg (2014). "Net reclassification improvement: computation, interpretation, and controversies: a literature review and clinician's guide". In: Annals of internal medicine 160.2, pp. 122-131.

Li, Lisha, Kevin Jamieson, Giulia DeSalvo, Afshin Rostamizadeh, and Ameet Talwalkar (2017). "Hyperband: A novel bandit-based approach to hyperparameter optimization". In: The Journal of Machine Learning Research 18.1, pp. 6765-6816.

Lichtenstein, Sarah, Baruch Fischhoff, and Lawrence D Phillips (1981). Calibration of probabilities: The state of the art to 1980. Tech. rep. Decision Research. Eugene, OR.

Mark, Dustin G, Jie Huang, Uli Chettipally, Mamata V Kene, Megan L Anderson, Erik P Hess, Dustin W Ballard, David R Vinson, and Mary E Reed (2018). "Performance of coronary risk scores 
among patients with chest pain in the emergency department". In: Journal of the American College of Cardiology 71.6, pp. 606-616.

Molnar, Christoph (2020). Interpretable Machine Learning. Lulu. com.

Moons, Karel GM, Andre Pascal Kengne, Diederick E Grobbee, Patrick Royston, Yvonne Vergouwe, Douglas G Altman, and Mark Woodward (2012). "Risk prediction models: II. External validation, model updating, and impact assessment". In: Heart 98.9, pp. 691-698.

Murphy, Allan H and Robert L Winkler (1977). "Reliability of subjective probability forecasts of precipitation and temperature". In: Journal of the Royal Statistical Society: Series C (Applied Statistics) 26.1, pp. 41-47.

Pepe, Margaret S, Jing Fan, Ziding Feng, Thomas Gerds, and Jorgen Hilden (2015). "The net reclassification index (NRI): a misleading measure of prediction improvement even with independent test data sets". In: Statistics in biosciences 7.2 , pp. 282-295.

Probst, Philipp, Anne-Laure Boulesteix, and Bernd Bischl (2019). "Tunability: Importance of Hyperparameters of Machine Learning Algorithms." In: Journal of Machine Learning Research 20.53, pp. $1-32$.

Probst, Philipp, Marvin N Wright, and Anne-Laure Boulesteix (2019). "Hyperparameters and tuning strategies for random forest". In: Wiley Interdisciplinary Reviews: Data Mining and Knowledge Discovery 9.3, e1301.

Roberts, David R, Volker Bahn, Simone Ciuti, Mark S Boyce, Jane Elith, Gurutzeta Guillera-Arroita, Severin Hauenstein, José J Lahoz-Monfort, Boris Schröder, Wilfried Thuiller, et al. (2017). "Cross-validation strategies for data with temporal, spatial, hierarchical, or phylogenetic structure". In: Ecography 40.8, pp. 913-929.

Rozenholc, Yves, Thoralf Mildenberger, and Ursula Gather (2010). "Combining regular and irregular histograms by penalized likelihood". In: Computational Statistics 83 Data Analysis 54.12, pp. 3313-3323.

Rui, P, K Kang, and JJ. Ashman (2016). National Hospital Ambulatory Medical Care Survey: 2016 emergency department summary tables. URL: https://www.cdc.gov/nchs/data/ahcd/nhamcs_emergency/2016_ed_web_tables.pdf.

Sabbatini, Amber K, Brahmajee K Nallamothu, and Keith E Kocher (2014). "Reducing variation in hospital admissions from the emergency department for low-mortality conditions may produce savings". In: Health affairs 33.9, pp. 1655-1663.

Saito, Takaya and Marc Rehmsmeier (2015). "The precision-recall plot is more informative than the ROC plot when evaluating binary classifiers on imbalanced datasets". In: PloS one 10.3.

Sanders, Frederick (1963). "On subjective probability forecasting". In: Journal of Applied Meteorology 2.2, pp. 191-201.

Scheinker, David and Margaret L Brandeau (2020). "Implementing analytics projects in a hospital: successes, failures, and opportunities". In: INFORMS Journal on Applied Analytics 50.3, pp. 176-189.

Schuler, Alejandro, Vincent Liu, Joe Wan, Alison Callahan, Madeleine Udell, David E Stark, and Nigam H Shah (2016). "Discovering patient phenotypes using generalized low rank models". In: Biocomputing 2016: Proceedings of the Pacific Symposium. World Scientific, pp. 144-155.

Scirica, Benjamin M (2010). "Acute coronary syndrome: emerging tools for diagnosis and risk assessment". In: Journal of the American College of Cardiology 55.14, pp. 1403-1415.

Segal, Mark and Yuanyuan Xiao (2011). "Multivariate random forests". In: Wiley Interdisciplinary Reviews: Data Mining and Knowledge Discovery 1.1, pp. 80-87.

Sendak, Mark P, William Ratliff, Dina Sarro, Elizabeth Alderton, Joseph Futoma, Michael Gao, Marshall Nichols, Mike Revoir, Faraz Yashar, Corinne Miller, et al. (2020). "Real-world integration of a sepsis deep learning technology into routine clinical care: implementation study". In: JMIR medical informatics 8.7, e15182.

Senn, Stephen (2005). "Dichotomania: an obsessive compulsive disorder that is badly affecting the quality of analysis of pharmaceutical trials". In: Proceedings of the International Statistical Institute, 55th Session, Sydney.

Sisk, Rose, Lijing Lin, Matthew Sperrin, Jessica K Barrett, Brian Tom, Karla Diaz-Ordaz, Niels Peek, and Glen P Martin (2021). "Informative presence and observation in routine health data: A review of methodology for clinical risk prediction". In: Journal of the American Medical Informatics Association 28.1, pp. $155-166$. 
Smith, Jennifer N, Jenna M Negrelli, Megha B Manek, Emily M Hawes, and Anthony J Viera (2015). "Diagnosis and management of acute coronary syndrome: an evidence-based update". In: The Journal of the American Board of Family Medicine 28.2, pp. 283-293.

Sperrin, Matthew, Glen P Martin, Rose Sisk, and Niels Peek (2020). "Missing data should be handled differently for prediction than for description or causal explanation". In: Journal of Clinical Epidemiology 125, pp. 183-187.

Steyerberg, Ewout W, Frank E Harrell Jr, Gerard JJM Borsboom, MJC Eijkemans, Yvonne Vergouwe, and J Dik F Habbema (2001). "Internal validation of predictive models: efficiency of some procedures for logistic regression analysis". In: Journal of clinical epidemiology 54.8, pp. 774-781.

Steyerberg, Ewout W, Andrew J Vickers, Nancy R Cook, Thomas Gerds, Mithat Gonen, Nancy Obuchowski, Michael J Pencina, and Michael W Kattan (2010). "Assessing the performance of prediction models: a framework for some traditional and novel measures". In: Epidemiology (Cambridge, Mass.) 21.1, p. 128.

Strobl, Carolin, Anne-Laure Boulesteix, Achim Zeileis, and Torsten Hothorn (2007). "Bias in random forest variable importance measures: Illustrations, sources and a solution". In: BMC bioinformatics 8.1, pp. 1-21.

Than, Martin, Dylan Flaws, Sharon Sanders, Jenny Doust, Paul Glasziou, Jeffery Kline, Sally Aldous, Richard Troughton, Christopher Reid, William A Parsonage, et al. (2014). "Development and validation of the E mergency D epartment A ssessment of $\mathrm{C}$ hest pain $\mathrm{S}$ core and $2 \mathrm{~h}$ accelerated diagnostic protocol". In: Emergency Medicine Australasia 26.1, pp. 34-44.

Than, Martin, Mel Herbert, Dylan Flaws, Louise Cullen, Erik Hess, Judd E Hollander, Deborah Diercks, Michael W Ardagh, Jeffery A Kline, Zea Munro, et al. (2013). "What is an acceptable risk of major adverse cardiac event in chest pain patients soon after discharge from the Emergency Department?: a clinical survey". In: International journal of cardiology 166.3, pp. 752-754.

Tibshirani, Robert (1996). "Regression shrinkage and selection via the lasso". In: Journal of the Royal Statistical Society: Series B (Methodological) 58.1, pp. 267-288.

Udell, Madeleine, Corinne Horn, Reza Zadeh, Stephen Boyd, et al. (2016). "Generalized low rank models". In: Foundations and Trends® in Machine Learning 9.1, pp. 1-118.

Van Calster, Ben, David J McLernon, Maarten van Smeden, Laure Wynants, and Ewout W Steyerberg (2019). "Calibration: the Achilles heel of predictive analytics". In: BMC medicine 17.1, pp. 1-7.

van der Laan, Mark J and Sandrine Dudoit (2003). "Unified cross-validation methodology for selection among estimators and a general cross-validated adaptive epsilon-net estimator: Finite sample oracle inequalities and examples". In:

van der Laan, Mark J, Eric C Polley, and Alan E Hubbard (2007). "Super learner". In: Statistical applications in genetics and molecular biology 6.1.

van Smeden, Maarten, Karel GM Moons, Joris AH de Groot, Gary S Collins, Douglas G Altman, Marinus JC Eijkemans, and Johannes B Reitsma (2019). "Sample size for binary logistic prediction models: beyond events per variable criteria". In: Statistical methods in medical research 28.8, pp. 2455-2474.

Vickers, Andrew J and Elena B Elkin (2006). "Decision curve analysis: a novel method for evaluating prediction models". In: Medical Decision Making 26.6, pp. 565-574.

Vickers, Andrew J, Ben Van Calster, and Ewout W Steyerberg (2016). "Net benefit approaches to the evaluation of prediction models, molecular markers, and diagnostic tests". In: $b m j 352$.

Williamson, Brian D, Peter B Gilbert, Noah Simon, and Marco Carone (2017). "Nonparametric variable importance assessment using machine learning techniques". In:

Wolpert, David H (1992). "Stacked generalization". In: Neural networks 5.2, pp. 241-259.

Wood, Simon N (2003). "Thin plate regression splines". In: Journal of the Royal Statistical Society: Series B (Statistical Methodology) 65.1, pp. 95-114.

Yates, J Frank (1982). "External correspondence: Decompositions of the mean probability score". In: Organizational Behavior and Human Performance 30.1, pp. 132-156. 